\title{
High-Intensity-Laser-Electron Scattering
}

\author{
David D. Meyerhofer
}

(Invited Paper)

\begin{abstract}
In the field of an intense laser, photon-electron scattering becomes nonlinear when the oscillatory energy of the electron approaches its rest mass. The electron wave function is dressed by the field with a concomitant increase in the effective electron mass. When the photon energy in the electron rest frame is comparable to the electron rest mass, multiphoton Compton scattering occurs. When the photon energy is significantly lower than the electron rest mass, the electron acquires momentum from the photon field and emits harmonics. This paper reviews nonlinear photon-electron scattering processes and results from two recent experiments where they have been observed.
\end{abstract}

Index Terms - Electromagnetic scattering, high intensity, multiphoton.

\section{INTRODUCTION}

$\mathbf{T}$ HE interaction (scattering) of light with free electrons is a well-known phenomenon. Thomson scattering, the scattering of an electromagnetic wave from an electron under the condition that the photon energy is much less than the electron rest mass, $\hbar \omega \ll m_{e} c^{2}$, was discovered in the late 19th century [1]. Compton scattering, the scattering of a photon and electron under the conditions where the photon energy in the electron rest frame is large enough that electron recoil effects must be included, was discovered in 1923 [2], and the kinematics were described in 1927 [3]. The scattering of two photons to produce an electron-positron pair was calculated by Breit and Wheeler in 1934 [4]. All of these processes are linear. In Thomson scattering, the electron response to the field is harmonic. In Compton and Breit-Wheeler processes, the scattering rates depend linearly on the photon fluxes. Recent advances in high-intensity-laser systems [5], [6] have allowed the study of the nonlinear analogues to the scattering processes described above. Nonlinear Thomson [7] and nonlinear Compton [8] scattering have been observed, and an experiment is underway to study nonlinear Breit-Wheeler scattering [9]. In Section II, nonlinear laser-electron scattering is described, both in the Thomson and Compton regimes. Nonlinear photon-photon scattering is described in Section III. Experimental measurements of nonlinear laser-electron scattering are reviewed briefly in

Manuscript received August 8, 1996; revised May 19, 1997. This work was supported by the National Science Foundation and by the U.S. Department of Energy (DOE) Office of Inertial Confinement Fusion under Cooperative Agreement DE-FC03-92SF19460, the University of Rochester, and the New York State Energy Research and Development Authority.

The author is with the Department of Mechanical Engineering, the Department of Physics and Astronomy, and the Laboratory for Laser Energetics, University of Rochester, Rochester, NY 14623 USA.

Publisher Item Identifier S 0018-9197(97)07829-9.
Sections IV and V for Thomson and Compton scattering, respectively. The conclusions are presented in Section VI.

\section{NONLINEAR LASER-ElECTRON SCATTERING}

The interaction of an electron with an intense laser field is characterized by [10]

$$
\eta^{2}=\frac{e^{2} E_{\mathrm{rms}}^{2}}{m_{e}^{2} \omega_{L}^{2} c^{2}}=-\frac{e^{2}}{m^{2} c^{4}}\left\langle A_{\mu} A^{\mu}\right\rangle
$$

where $E_{\mathrm{rms}}$ is the rms electric field strength, $\omega_{L}$ is the laser frequency, and $A_{\mu}$ is the four-vector potential. As defined in (1), $\eta^{2}$ has the same value for linear and circular polarization at a given laser intensity. For counterpropagating electron and photon beams, $\eta^{2}$ is an invariant, independent of the electron beam energy in the laboratory.

In 1929, Volkov calculated the electron wave function in an electromagnetic wave [11]. One can interpret these wave functions as dressed states of an electron in an electromagnetic field. The electron's rest mass is dressed by the field giving an effective mass, $m^{* 2}=m_{0}^{2}\left(1+\eta^{2}\right)$. At low intensities $\eta^{2} \ll 1, \eta$ is equal to the ratio of the quiver velocity in the field to the speed of light, and $\eta^{2} / 2$ is the ratio of the average oscillatory energy to the rest mass. $\eta^{2}$ is directly related to the ponderomotive potential [12], [13],

$$
\Phi_{\text {pond }}=\frac{e^{2} E_{\mathrm{rms}}^{2}}{2 m \omega^{2}}=\frac{\eta^{2}}{2} m_{e} c^{2}
$$

and has the numerical value $\eta^{2}=3.6 \times 10^{-19} I$ $\left(\mathrm{W} / \mathrm{cm}^{2}\right) \lambda_{L}^{2}(\mu \mathrm{m})$. For $1-\mu \mathrm{m}$-wavelength light, $\eta^{2}$ approaches 1 as the intensity approaches $10^{18} \mathrm{~W} / \mathrm{cm}^{2}$.

In a quantum mechanical picture, multiphoton-electron scattering processes can be calculated as transitions between dressed states [14]-[16]. Many other quantum mechanical calculations have been carried out. Classically, intense field interactions with electron can be calculated as anharmonic corrections to the electron trajectory, which become prominent as $\eta^{2}$ approaches 1 [17]-[20].

The distinction between quantum mechanical and classical descriptions of the interactions of an electron with an intense field is made for convenience. For example, the Volkov states [11] include the classical electron trajectory in the calculation of the wave function. Similarly, the distinction between Thomson and Compton scattering is somewhat arbitrary. The kinematics of multiphoton-electron scattering can be used to explore the different regimes.

An $n$ photon-electron scattering process can represent either a single interaction of $n$ photons with an electron (multiphoton 

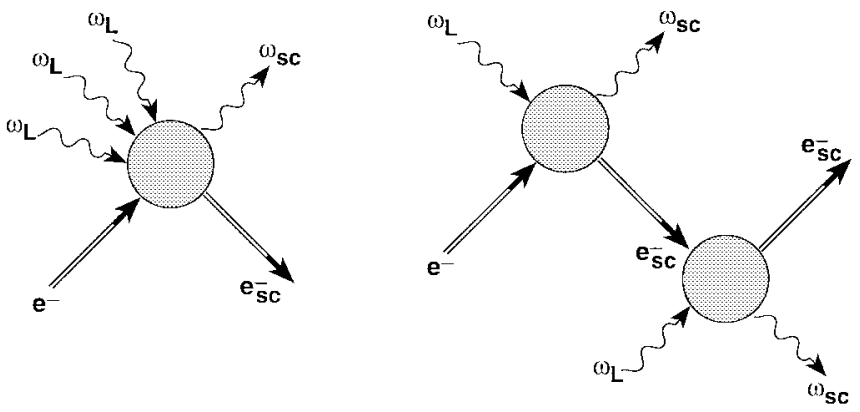

Fig. 1. Diagrams showing multiphoton-electron scattering and plural scattering. The electrons are represented as double lines and the vertices as circles to indicate that the scattering is taking place in the presence of a strong field and that the electron-wave function is dressed by the field.

or nonlinear scattering), or a series of single photon-electron scattering events (plural scattering), or a combination thereof [8]. The electron kinematics of these two process are almost identical. Fig. 1 shows a representative diagram of the two scattering processes. In Fig. 1, the electrons are represented as double lines and the vertices as circles to indicate that the scattering takes place in the presence of a strong field and that the electron wave function is dressed by the field. A nonlinear, $n$ photon-electron scattering event can be described as a single interaction

$$
e^{-}+n \omega_{L} \rightarrow e^{\prime-}+\omega_{\mathrm{sc}}
$$

while a plural scattering event can be described as a sequence of interactions

$$
\begin{gathered}
e^{-}+\omega_{L} \rightarrow e^{\prime-}+\omega_{\mathrm{sc}} \\
e^{\prime-}+\omega_{L} \rightarrow e^{\prime \prime}+\omega_{\mathrm{sc}}^{\prime} \\
e^{\prime \prime-}+\omega_{L} \rightarrow e^{\prime \prime \prime}+\omega_{\mathrm{sc}}^{\prime \prime}
\end{gathered}
$$

The kinematics of the two processes are almost identical, and the scattered electron energies for counterpropagating electron and photon beams in the laboratory frame can be written

$$
\begin{aligned}
E_{\mathrm{sc}}= & \gamma m c^{2}+n \hbar \omega_{L} \\
& -\frac{2(1+\beta) n \hbar \omega_{L} \gamma^{2}}{2 \gamma^{2}(1-\beta \cos \theta)+\left(\frac{2 n \hbar \omega \gamma}{m c^{2}}+\frac{\eta^{2}}{1+\beta}\right)(1+\cos \theta)}
\end{aligned}
$$

where $\gamma m_{e} c^{2}$ is the initial electron energy with a corresponding velocity parameter, $\beta \gamma=\sqrt{\gamma^{2}-1}$. The incident photon energy is $\hbar \omega_{L}$, and $\theta$ is the laboratory scattering angle of the photon, where $\theta=0$ corresponds to backscattering of the photon. Equation (5) describes the scattered electron energy for a nonlinear, $n$-photon scattering event and is a reasonable approximation for $n$-photon plural scattering. The scatteredelectron energy can be greater or less than the initial electron energy depending on the experimental conditions.
The scattered photon energy for a nonlinear, $n$-photon scattering event is

$$
\hbar \omega_{\mathrm{sc}}=\frac{2(1+\beta) n \hbar \omega_{L} \gamma^{2}}{2 \gamma^{2}(1-\beta \cos \theta)+\left(\frac{2 n \hbar \omega \gamma}{m c^{2}}+\frac{\eta^{2}}{1+\beta}\right)(1+\cos \theta)} .
$$

Equations (5) and (6) reduce to the well-known Klein-Nishina formula [3] as $\eta \rightarrow 0$ and $n \rightarrow 1$ and to the standard Compton formula as, in addition, $\beta \rightarrow 0$ and $\gamma \rightarrow 1$.

One can discuss the relationship between Thomson and Compton scattering by examining the energy change of either the photon or electron during the scattering process. If we first consider the scattering of a single photon from an electron at rest $(\gamma=1)$ at low intensities, $\eta \sim 0$, the energy change is

$$
\hbar \omega_{L}-\hbar \omega_{\mathrm{sc}}=-\Delta E_{e}=\frac{\hbar \omega_{L}}{m_{e} c^{2}} \frac{1-\cos \theta}{1-\frac{\hbar \omega_{L}}{m_{e} c^{2}}(1-\cos \theta)} \hbar \omega_{L} .
$$

Compton's original scattering experiment [2] was carried out with Mo K- $\alpha$ photons with energy of approximately $17 \mathrm{keV}$ interacting with electrons at rest, so $\hbar \omega / m_{e} c^{2} \sim 3 \%$ and the observed frequency shifts of scattered light were of the same order of magnitude. Recoil electrons with energies of a few percent of the incident photon energy were subsequently observed.

Thomson scattering traditionally refers to the scattering of visible or longer-wavelength light by free electrons with $\gamma \sim 1$ [21]. Under this condition, $\hbar \omega / m_{e} c^{2} \ll 1$, the changes in photon and electron energy are ignored in the scattering process. Thomson scattering can be described by treating the photons as an electromagnetic wave, determining the electron response to the wave and the subsequent radiation [21]. This classical picture of the electron's response is discussed below.

Traditionally the distinction between Thomson and Compton scattering has been that for Thomson scattering the electron recoil and photon frequency shift can be ignored because $\gamma \hbar \omega / m_{e} c^{2} \ll 1$, while for Compton scattering, the photon energy in the rest frame of the electron, $\gamma \hbar \omega / m_{e} c^{2}$, is large enough that the electron recoil and photon frequency shift are nonnegligible. While this distinction is reasonable when single-photon scattering is considered, it becomes less clear when multiple-photon-electron scattering occurs. In this case, the more relevant parameter is $\gamma n \hbar \omega / m_{e} c^{2}$. In the Thomson regime, with $\gamma \hbar \omega / m_{e} c^{2} \ll 1$, sufficiently large numbers of photons can participate in the interaction to make the electron recoil and photon frequency shift nonnegligible. For an electron initially at rest, the scattered-electron energy for an $n$-photon scattering is approximately

$$
\Delta E_{e} \approx \frac{n \hbar \omega_{L}}{m_{e} c^{2}} \frac{n \hbar \omega_{L}}{1+\frac{n \hbar \omega_{L}}{m_{e} c^{2}}}
$$

Thus, if sufficiently large numbers of photons are scattered, the electron can gain an energy comparable to its rest mass. This becomes possible as $\eta^{2}$ approaches 1. Equation (8) is equally valid for nonlinear and plural scattering.

In the traditionally Compton regime, the probability of multiphoton, or nonlinear, Compton scattering

$$
e^{-}+n \omega \rightarrow e^{-}+\omega_{\mathrm{sc}}
$$


scales approximately as $P_{n} \propto \eta^{2 n}[16]$. As $\eta^{2}$ becomes large enough to cause significant scattering, the dressing (shift) of the electron rest mass by the electromagnetic field, $m^{*}=$ $m_{0} \sqrt{1+\eta^{2}}$, cannot be ignored. The scattered-photon energy can be related to the incident photon energy for photons colliding with electrons with energy $\gamma m_{0} c^{2}$. The maximum backscattered photon energy for head-on collisions is [10]

$$
\hbar \omega_{\mathrm{sc}}=\frac{4 n \hbar \omega_{L} \gamma^{2}}{1+\frac{4 n \gamma \hbar \omega_{L}}{m_{e} c^{2}}+\eta^{2}}
$$

which corresponds to a minimum scattered-electron energy

$$
E_{\mathrm{sc}}=\frac{\gamma m_{e} c^{2}\left(1+\eta^{2}\right)}{1+\frac{4 n \gamma \hbar \omega_{L}}{m_{e} c^{2}}+\eta^{2}}
$$

when the photon energy in the laboratory frame is much less than the electron rest energy. Thus, $\eta^{2}$ enters both in the rate of multiphoton emission and in causing a shift in the scattered photon frequency due to the mass shift. The Thomson-scattering process, both cross-section and kinematics, is recovered as the low-energy limit of the Comptonscattering cross section [10].

When the photon energy in the electron rest frame is much less than the electron rest mass, the laser can be treated as an electromagnetic field and the laser-electron interaction can be described classically. When the photon energy in the electron rest frame is comparable to the electron rest mass, the interaction must be treated quantum mechanically and the photon nature of the field must be considered.

The transition to multiphoton-electron scattering in a highintensity laser focus occurs when the oscillatory energy of a free electron in the laser field approaches the electron rest energy. Under these conditions, the electron recoil due to the laser field momentum can no longer be ignored, and free electrons acquire momentum in the direction of the wavevector of the laser. This momentum was predicted from both quantum mechanical [22]-[26] and classical considerations [19], [20] in the 1960's and in a series of later calculations [27]-[33].

In the Thomson regime $\left(\hbar \omega \ll m_{e} c^{2}\right)$, where the photon energy is much less than the electron rest energy, harmonic emission and a mass shift of the electrons are also governed by $\eta^{2}$ [22]-[26]. Under these conditions, the mass shift can be thought of as a Doppler shift associated with the forward momentum of the electrons in the field [25]. The forward momentum arises from conservation of energy and momentum during the dressing of the electron.

An electron in a plane wave is accelerated by the Lorentz force

$$
\frac{d \vec{p}}{d t}=-e\left[\frac{1}{c} \frac{\partial \vec{A}}{\partial t}+\frac{\vec{p} \times(\vec{\nabla} \times \vec{A})}{\gamma m c}\right] .
$$

The Lorentz-force equation can be solved by separating it into an equation in the plane of polarization and the $\vec{k}(\hat{z})$ direction. The solution of these equations, for an electron initially at rest, is [34]

$$
\begin{aligned}
\vec{p}_{\perp} & =\gamma m c \vec{\beta}_{\perp}=\frac{e}{c}[\vec{A}(t)-\vec{A}(0)] \\
p_{\vec{k}} & =\gamma m c \beta_{\vec{k}}=m c(\gamma-1) \\
\gamma m c^{2} & =m c^{2}+\frac{1}{2} \frac{e^{2}}{m c^{2}}[\vec{A}(t)-\vec{A}(0)]^{2}
\end{aligned}
$$

where $\vec{\beta}=\vec{v} / c$ and $\vec{A}(0)$ is the initial vector potential. In this case, the parallel direction is that of the $k$ vector of the laser, and the perpendicular direction is in the plane of polarization. The mass shift of the electron in the field is given by the time average of the equation for $\gamma m c^{2}$. For linearly polarized laser light with, for example, $\vec{A}(t)=A_{0} \sin \omega t \hat{x}$, (13) yields a trajectory that includes anharmonic motion of the electron in the field and a forward drift. The anharmonic motion is a figure- 8 motion in the plane made by the laser polarization and $k$ vector for linear polarization [18], [20], [34]. The nonlinear motion leads to the radiation of harmonics from the laser [17], [19], [20], while the forward motion leads to a wavelength shift of the harmonics as shown in the quantum mechanical calculations [25]. This wavelength shift is equivalent to the quantum mechanical mass shift in the field [25]. The rate of harmonic emission scales approximately as $R_{n} \propto \eta^{2 n}$ [17].

In circular polarization, the trajectory is circular in the plane of polarization with an angular frequency $\omega_{L}$, and radius

$$
r=\frac{\eta}{\sqrt{1+\eta^{2}}} \frac{c}{\omega_{L}} .
$$

The electron also undergoes a drift in the $\vec{k}$ direction of the laser. Harmonics of the laser light are observed due to the modulation of the distance from the radiating electron to the observer [20].

Conservation of momentum and energy during the photonscattering process can be used to derive the relationship between the perpendicular and parallel momenta in (13) [28], [29], [35]. If the electron gains an energy $\Delta E_{e}$ from the photon field, it also gains a longitudinal ( $\vec{k}$ direction) momentum $c p_{\vec{k}}=\Delta E_{e}$. Thus, if an electron, initially at rest, gains an energy $\Delta E_{e}=(\gamma-1) m_{e} c^{2}$, its final parallel momentum will be related to its perpendicular momentum by [28], [29], [35]

$$
p_{\vec{k}}=\frac{p_{\perp}^{2}}{2 m_{0} c} .
$$

When the electron is in the laser field, the perpendicular momentum is due to oscillatory motion in the field.

In a laser focus, the electron feels a ponderomotive force $\vec{F}_{p}=-\vec{\nabla} \Phi_{\text {pond }}$, which causes the electron to be ejected from the laser focus with a directed kinetic energy equal to its oscillatory energy in the field [12]. The angle of the ejected electron relative to the wavevector of the laser $(\theta)$ depends on the final electron energy $\gamma m c^{2}$ as [28], [29], [35]

$$
\tan \theta=\sqrt{\frac{2}{\gamma-1}} .
$$

Thus, for low intensities, the electron is ejected with an energy much less than its rest mass nearly perpendicular to the $\vec{k}$ direction. In ultrarelativistic conditions $\gamma \gg 1$, the electron leaves the laser focus almost parallel to the $\vec{k}$ direction. 
When an electron is incident on the focus of a laser, it can be scattered by the ponderomotive potential [12]. This effect was observed with low-energy electrons by Bucksbaum et al. [36].

In an intense field, the interaction of the laser pulse with free electrons yields shifted harmonics of the laser frequency. This is true in both the Compton and Thomson regimes, though in the Compton regime, the scattered-photon energy can be much higher in the laboratory frame than in the rest frame of the electron [10].

In 1983, Englert and Reinhart [37] reported a preliminary observation of second-harmonic emission from the scattering of a moderate-intensity, multimode laser from a low-energy electron beam. They observed second-harmonic photons with the expected Doppler shift associated with the electron beam. The observed rates were extremely low but appeared to be consistent with predictions [17], [20].

Results from two recent multiphoton-electron scattering experiments $\left(\eta^{2} \sim 1\right)$ have been reported. In one experiment, the electrons are initially at rest in the laboratory frame [7], while in the other, $50-\mathrm{GeV}$ electrons produced at the Stanford Linear Accelerator Center collide with a counterpropagating laser beam [8]. In both cases, nonlinear photon-electron scattering has been observed. These experiments are briefly described in Sections IV and V.

\section{NONLINEAR PHOTON-PHOTON SCATTERING}

The rate of electron-positron pair production by photon-photon scattering was calculated by Breit and Wheeler in 1934 [4]. For pair production with counterpropagating photons, the product of the photon energies must be greater than the pair mass squared, $\hbar \omega_{1} \hbar \omega_{2} \geq m_{e}^{2} c^{4}$. When the laser field $E_{L}$ approaches the QED critical field [38], $E_{\text {crit }}$, nonlinear photon-photon scattering

$$
\omega_{1}+n \omega_{2} \rightarrow e^{-}+e^{+}
$$

becomes possible [10], [15], [16], [39]. The photon-energy requirement for nonlinear pair production becomes

$$
\hbar \omega_{1} n \hbar \omega_{2} \geq m_{e}^{* 2} c^{4}
$$

where $m_{e}^{*}$ is the electron mass dressed by the field. The QED critical field [38] corresponds to the electric-field strength, where an electron gains an energy equal to its rest mass in a Compton wavelength

$$
e E_{\mathrm{cr}} \lambda_{C}=m_{e} c^{2}
$$

and is equal to $E_{\mathrm{cr}}=m_{e}^{2} c^{2} / e \hbar=1.3 \times 10^{16} \mathrm{~V} / \mathrm{cm}$. The QED critical field corresponds also to binding electric field of the ground state of a hydrogenic ion with nuclear charge equal to 137.

The QED critical field corresponds to a laser intensity of $4 \times 10^{29} \mathrm{~W} / \mathrm{cm}^{2}$, well beyond the reach of current laser technology [6]. This field strength can be reached, however, in the rest frame of an energetic electron because the laser field transforms as $E_{\text {rest }}=\gamma(1+\beta) E_{\text {lab }}$ for counterpropagating electron and laser beams. The QED critical-field strength can also be reached in the center of momentum frame associated with the collisions of photons of different energies. For a

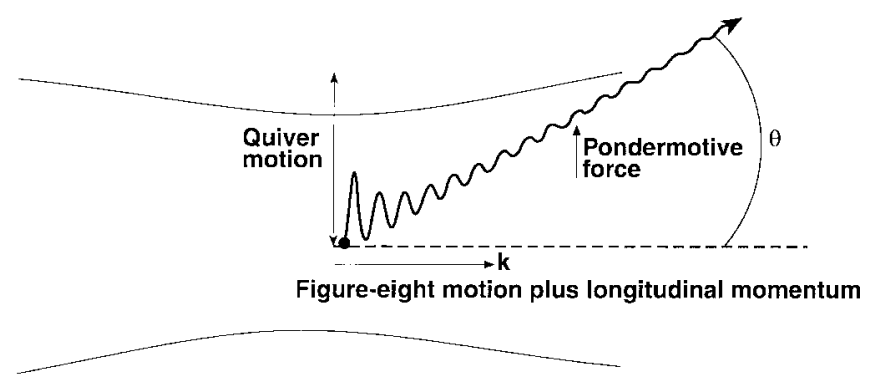

Fig. 2. Schematic figure of the experimental interaction region where the scattering of low-energy electrons born by ionization in a laser focus by an intense laser is observed. Electrons are observed with forward momentum due to conservation of photon momentum.

collision of a high-energy photon $\omega_{1}$ with an intense laser, the field strength in the center of momentum frame is

$$
E_{\mathrm{com}}=\sqrt{\frac{\omega_{1}}{\omega_{L}}} E_{L}
$$

Thus nonlinear photon-photon scattering may be observed during the scattering of an intense laser with energetic electrons in a two-step process [9], [39]. High-energy gammas produced by Compton scattering can subsequently interact with the laser field, leading to the production of electron positron pairs. The ratio of the laser field in the rest frame of the electron is to the QED critical field has been given the symbol upsilon, $Y=E_{\text {rest }} / E_{\mathrm{cr}}$.

\section{NONLINEAR LASER-ELECTRON SCATTERING IN THE THOMSON REGIME}

When electrons are born at rest through field ionization in a high-intensity-laser focus, they acquire energy and momentum from the laser field through their quiver energy in the field and conservation of momentum. As the electron's kinetic energy approaches its rest energy, the absorption of momentum from the field cannot be ignored and the electrons gain forward momentum. In [7] the experimental observation of electron recoil during high-intensity-laser-electron scattering (Compton scattering) in the Thomson regime with photon energy much less than the electron rest mass, $\hbar \omega \ll m_{e} c^{2}$, was described. A particularly simple relationship between perpendicular and parallel momentum derived from conservation of energy and momentum in the field was shown in (15). In this low-electronenergy experiment, electrons were injected into the field at rest during the ionization of $\mathrm{Ne}$ and $\mathrm{Kr}$ atoms and ions by a high-intensity laser [7], [40]. The electrons gained both energy and longitudinal momentum from the field. They were subsequently accelerated out of the focus by the ponderomotive force, retaining their longitudinal momentum. A schematic of the experimental interaction is shown in Fig. 2. Electrons born by field ionization undergo oscillatory motion plus a drift in the $\vec{k}$ direction. The ponderomotive force connects the oscillatory energy to directed kinetic energy and the electrons escape the focus with both practical and longitudinal momentum.

A magnetic spectrometer was used to measure the energy and angular (relative to $\vec{k}$ ) distributions of electrons emitted from a high-intensity-laser focus [41]. The spectrometer consists of an energy-resolving magnet and a detector consisting 
of a scintillator coupled to a photomultiplier tube (PMT). The magnet lies in a plane above the focus with an energyangular resolving gap. The central axis of the spectrometer is perpendicular to the $\vec{k}$-direction of the laser and passes through the laser focus. The angular distribution of electrons relative to the laser $\vec{k}(\theta)$ is measured by rotating the spectrometer about its central axis $(\phi)$. The energy is resolved by varying the magnetic field in the gap. The energy window of the spectrometer was varied by changing the magnetic field in the gap of the steering magnet and was calibrated using an electron gun placed at the laser focus and aimed toward the gap in the steering magnet. The calibration showed an energy window of $\Delta E / E \sim 0.3$ FWHM.

The angular distribution of electrons in $\theta$ (relative to $\vec{k}$ ) is measured by rotating the entire spectrometer about the cylindrical axis that passes through the laser focus at $90^{\circ}$ to the laser axis. The gap in the magnet is offset from the central axis of the spectrometer and is always aligned so that a clear line of sight can be traced from anywhere on this central axis through the gap in the magnet. The angular resolution is $\pm 1.5^{\circ}$. The experiments were conducted with a $1.053-\mu \mathrm{m}$, 1-ps laser using chirped-pulse amplification (CPA) [42]. The laser is focused with $f / 3$ optics producing a $5-\mu \mathrm{m}\left(1 / e^{-2}\right.$ radius) focal spot and a peak laser intensity of approximately $10^{18} \mathrm{~W} / \mathrm{cm}^{2}$ into neon and krypton at a pressure of $10^{-3}$ torr with circular polarization.

In experiments by Moore et al. [7], the angular and energy distributions of electrons injected into the field during the field ionization [43] of $\mathrm{Ne}$ and $\mathrm{Kr}$ were measured. Electrons associated with $\mathrm{Ne}^{8+}$ emerged from the circularly polarized focus with an energy of $80 \pm 5 \mathrm{keV}$ and at an angle of $75 \pm 1.5^{\circ}$ from the laser axis ( $\vec{k}$ direction). These results were extended to the observation of electrons injected during the production of $\mathrm{Kr}^{10+}$ and $\mathrm{Kr}^{11+}$ [40]. The energy and angular spectra are in good agreement with the predictions of calculations that include the relativistic mass shift. Fig. 3 shows the observed energy and angular distribution for $\mathrm{Ne}^{3+}-\mathrm{Ne}^{8+}$ and $\mathrm{Kr}^{10+}$, and $\mathrm{Kr}^{11+}$ (from [7] and [40]). In all cases, the observations are in good agreement with the relativistic calculations, with the energies predicted by classical field ionization [43] and subsequent ejection from the focus by ponderomotive and canonical momentum effects. The solid curve is that predicted by (16). The energy and angular spectra were in good agreement with the predictions of calculations that include the relativistic mass shift [7], [40]. For electrons from the highest charge states from $\mathrm{Kr}$, differences in the energy and angular distribution due to the electron mass shift in the field were observed [40].

\section{NONLINEAR LASER-ELECTRON SCATTERING IN THE COMPTON LIMIT}

Nonlinear Compton scattering of two to four laser photons has been reported during the interaction of a high-intensity laser $\left(I \sim 10^{18} \mathrm{~W} / \mathrm{cm}^{2}\right)$ counterpropagating with $47-\mathrm{GeV}$ electrons at the Stanford Linear Accelerator Center [8]. In this case, the photon energy in the electron rest frame is comparable to the electron rest mass $\left(\gamma \hbar \omega_{L} / m_{e} c^{2} \sim 1\right)$.

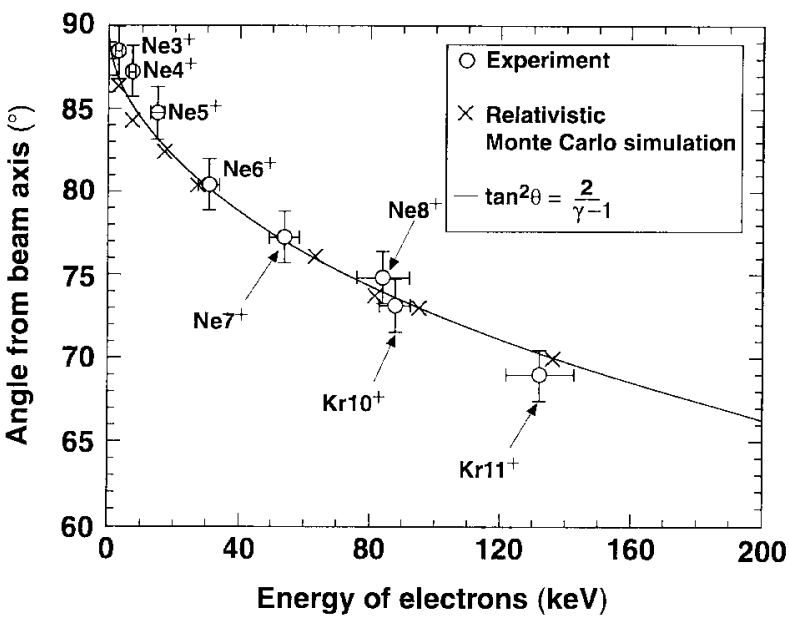

Fig. 3. Comparison of observed (with errors) and relativistic Monte Carlo calculations $(\mathrm{X})$ of the energy and ejection angle for electrons born during the production of various charge states of $\mathrm{Ne}$ and $\mathrm{Kr}$. Equation (15) is plotted for comparison. (From [40])

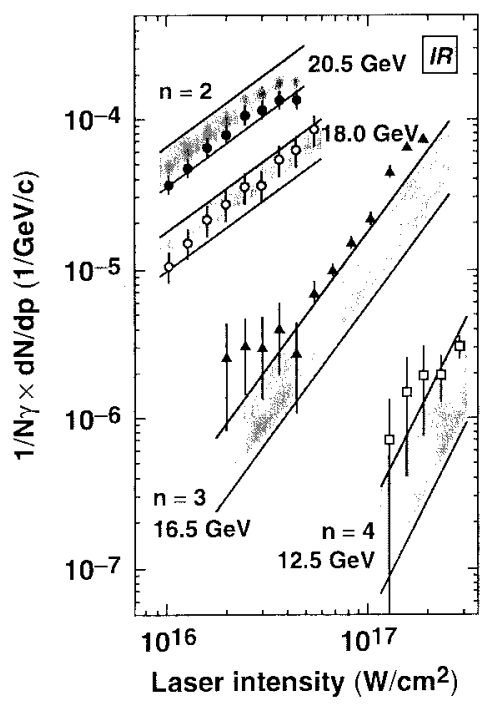

Fig. 4. The normalized yield of scattered electrons as a function of laser intensity for a variety of electron energies corresponding to $n=$ two-, three-, and four-photon nonlinear Compton scattering. The shaded areas are the theoretical predictions for the electron spectra determined by simulating the interaction using the rates from [10], [15], [16]. (From [8]).

The electron beam interacts with a $0.5-\mathrm{Hz}$-repetition-rate, $1-\mu \mathrm{m}, 1-\mathrm{ps}$, terawatt, CPA laser system using a flashlamppumped, Nd:glass, zigzag slab amplifier [44]. The laser produces infrared pulses with energies greater than $2 \mathrm{~J}$ with an average power in excess of $1 \mathrm{~W}$ and 1.4-times-diffractionlimited focusing. Frequency doubling of these pulses is accomplished with up to 55\% efficiency. The laser has been synchronized with a $47-\mathrm{GeV}$ electron beam at the Stanford Linear Accelerator with a temporal jitter of $\sigma=2$ ps between the two beams [45].

In the experiment, the scattered electrons are observed by passing them through a magnetic spectrometer and detecting them with segmented silicon calorimeters [8], [9]. The number of scattered electrons as a function of scattered energy is measured. The energy of the electrons undergoing multiphoton 
Compton scattering can be lower than those allowed for single-photon Compton scattering, as shown in (11). Thus, the existence of low-energy scattered electrons is evidence for multiphoton Compton scattering. For multiphoton orders higher than 2, plural Compton scattering is significantly less probable than multiphoton scattering. The experiment has observed nonlinear Compton scattering with up to four photons participating in a single scattering event. Fig. 4 shows the normalized yield of scattered electrons as a function of laser intensity for a variety of electron energies corresponding to $n=$ two-, three-, and four-photon nonlinear Compton scattering. The shaded areas are the theoretical predictions calculated by numerically simulating the interaction using the rates from [10], [15], [16]. The observed multiphoton Compton scattering cross sections were found be in good agreement with the nonlinear QED predictions for laser wavelengths of 1.053 and $0.527 \mu \mathrm{m}$ [8].

In this experiment, the laser field strength in the electron rest frame approaches the QED critical field corresponding to a laser intensity of approximately $10^{29} \mathrm{~W} / \mathrm{cm}^{2}, Y \sim 1$. Electron-positron pairs due to multiphoton-photon scattering can be created in a two-step process during this interaction [9]. After Compton scattering, the high-energy scattered photon $(\sim 30 \mathrm{GeV})$ can interact further with the laser field producing electron positron pairs via the process described in Section III. Positron production during multiphoton light by light scattering has been observed in these experiments [46].

\section{CONCLUSION}

In the focus of a high-intensity laser, photon-electron and photon-photon scattering processes become nonlinear. The electron's oscillatory energy in the field can exceed its rest mass. Recent experiments have observed nonlinear scattering processes during laser-electron scattering with low-energy electrons born at rest [7] and with 46.6-GeV electrons [8]. Studies of nonlinear inelastic photon-photon scattering are underway [9], [46]. In the past few years, advances in laser technology have begun to open up a new area of high-field studies where the traditional boundaries between Thomson and Compton scattering become less clear. For example, nonlinear Compton scattering of $n$ photons and an electron takes place in the presence of an intense laser field, mixing quantum mechanical and classical pictures.

\section{ACKNOWLEDGMENT}

The author thanks J. H. Eberly, S. Goreslavskii, J. P. Knauer, K. T. McDonald, A. C. Melissinos, and N. Narozhny for many stimulating discussions. The experiments on multiphoton-electron scattering with low-energy electrons were carried out in collaboration with C. I. Moore, J. P. Knauer, and S. J. McNaught. The nonlinear QED experiments at the Stanford Linear Accelerator Center were carried out by a collaboration between the University of Rochester, Princeton University, the University of Tennessee, and the Stanford Linear Accelerator Center.

\section{REFERENCES}

[1] J. J. Thomson, Notes on Recent Researches in Electricity and Magnetism. Oxford, U.K.: Clarendon Press, 1893

[2] A. H. Compton, "The spectrum of scattered x-rays," Phys. Rev., vol. 22, pp. 409-413, 1923.

[3] O. Klein and Y. Nishina, "Scattering of radiation by free electrons on the new relativistic quantum dynamics of dirac," Z. Phys., vol. 52, pp. 853-868, 1929.

[4] G. Breit and J. A. Wheeler, "Collision of two light quanta," Phys. Rev., vol. 46, pp. 1087-1091, 1934.

[5] P. Maine, D. Strickland, P. Bado, M. Pessot, and G. Mourou, "Generation of ultrahigh peak power pulses by chirped pulse amplification," IEEE J. Quantum Electron., vol. 24, pp. 398-403, 1988.

[6] M. D. Perry and G. Mourou, "Terawatt to petawatt subpicosecond lasers," Science, vol. 264, pp. 917-924, 1994.

[7] C. I. Moore, J. P. Knauer, and D. D. Meyerhofer, "Observation of the transition from Thomson to Compton scattering in multiphoton interactions with low-energy electrons," Phys. Rev. Lett., vol. 74, pp. 2439-2442, 1995.

[8] C. Bula, K. T. McDonald, E. J. Prebys, C. Bamber, S. Boege, T. Kotseroglou, A. C. Melissinos, D. D. Meyerhofer, W. Ragg, D. L. Burke, R. C. Field, G. Horton-Smith, A. C. Odian, J. E. Spencer, D. Walz, S. C. Berridge, W. M. Bugg, K. Shmakov, and A. W. Weidemann, "Observation of nonlinear effects in Compton scattering," Phys. Rev. Lett., vol. 76, pp. 3116-3119, 1996.

[9] C. Bula, C. Lu, K. T. McDonald, E. Prebys, C. Bamber, T. Blalock, S Boege, A. C. Melissinos, D. Meyerhofer, Th. Kotseroglou, T. Barklow, D. L. Burke, P. Chen, R. Field, A. C. Odian, S. H. Rokni, and J. E. Spencer, "Study of QED at critical field strength in intense laser-high energy electron collisions at the stanford linear accelerator," Princeton University, University of Rochester, Stanford Linear Accelerator Center, University of Tennessee SLAC experiment, E-144, 1992.

[10] V. B. Berestetskii, E. M. Lifshitz, and L. P. Pitaevski, Quantum Electrodynamics, 2nd ed. Oxford, U.K.: Pergamon, 1982, sect. 101.

[11] D. M. Volkov, "Über eine klasse von lösungen der diracschen gleichung," Z. Phys., vol. 94, pp. 250-260, 1929.

[12] H. A. H. Boot, S. A. Self, and R. B. R.-Shersby-Harvie, J. Electron Control, vol. IV, pp. 434-453, 1958.

[13] W. L. Kruer, The Physics of Laser Plasma Interactions, Frontiers in Physics. Redwood City, CA: Addison-Wesley, 1988.

[14] H. R. Reiss, "Absorption of light by light," J. Math. Phys., vol. 3, pp. 59-67, 1962.

[15] A. I. Nikishov and V. I. Ritus, "Quantum processes in the field of a plane electromagnetic wave and in a constant field. I," Sov. Phys. JETP, vol. 19 , pp. 529-541, 1964.

[16] N. B. Narozhnyi, A. I. Nikishov, and V. I. Ritus, "Quantum processes in the field of a circularly polarized electromagnetic wave," Sov. Phys. JETP, vol. 20, pp. 622-629, 1965.

[17] Vachaspati, "Harmonics in the scattering of light by free electrons," Phys. Rev., vol. 128, pp. 664-666, 1962.

[18] _ "Exact solution of relativistic equations of motion of an electron in an external radiation field," in Proc. Natl. Inst. Sci. India, 1962, vol. 29 , pp. $138-142$.

[19] J. H. Eberly and A. Sleeper, "Trajectory and mass shift of a classical electron in a radiation pulse," Phys. Rev., vol. 176, pp. 1570-1573, 1968.

[20] E. S. Sarachik and G. T. Schappert, "Classical theory of scattering of intense laser radiation by free electrons," Phys. Rev. D, vol. 1, pp 2738-2753, 1970.

[21] J. D. Jackson, Classical Electrodynamics. New York: Wiley, 1975.

[22] L. S. Brown and T. W. B. Kibble, "Interaction of intense laser beams with electrons," Phys. Rev., vol. 133, pp. A705-A719, 1964.

[23] O. von Roos, "Interaction of very intense radiation fields with atomic systems," Phys. Rev., vol. 135, pp. A43-A50, 1964.

[24] Z. Fried and J. H. Eberly, "Scattering of a high-intensity, low-frequency electromagnetic wave by an unbound electron," Phys. Rev., vol. 136, pp. B871-B887, 1964

[25] T. W. B. Kibble, "Mutual refraction of electrons and photons," Phys. Rev., vol. 150, pp. 1060-1069, 1966.

[26] J. H. Eberly, "Interaction of very intense light with free electrons," in Progress in Optics, E. Wolf, Ed. Amsterdam, The Netherlands: North-Holland, 1969, vol. 7, pp. 359-415.

[27] H. Hora and G. Viera, "The lateral injection free electron laser: Momentum balance and axial shift of electrons," in Laser Interaction and Related Plasma Phenomena, H. Hora and G. H. Miley, Eds. New York: Plenum, 1985, vol. 6, pp. 201-208.

[28] J. N. Bardsley, B. M. Penetrante, and M. H. Mittleman, "Relativistic dynamics of electrons in intense laser fields," Phys. Rev. A, vol. 40, pp. $3823-3835,1989$. 
[29] H. R. Reiss, "Relativistic strong-field photoionization," J. Opt. Soc. Amer. B, vol. 7, pp. 574-586, 1990.

[30] N. B. Delone and V. P. Krainov, "Energy and angular electron spectra for the tunnel ionization of atoms by strong low-frequency radiation," J. Opt. Soc. Amer. B, vol. 8, pp. 1207-1211, 1991.

[31] W. B. Mori and T. Katsouleas, "Ponderomotive force of a uniform electromagnetic wave in a time varying dielectric medium," Phys. Rev. Lett., vol. 69, pp. 3495-3498, 1992.

[32] R. Grobe and M. V. Fedorov, "Packet spreading, stabilization, and localization in superstrong fields-reply," Phys. Rev. Lett., vol. 70, pp. $1562-1562,1993$.

[33] F. V. Hartemann, S. N. Fochs, G. P. Le Sage, N. C. Luhmann, Jr., J. G. Woodworth, M. D. Perry, Y. J. Chen, and A. K. Kerman, "Nonlinear ponderomotive scattering of relativistic electrons by an intense laser field at focus," Phys. Rev. E, vol. 51, pp. 4833-4843, 1995.

[34] L. D. Landau and E. M. Lifshitz, The Classical Theory of Fields, Course of Theoretical Physics. Oxford, U.K.: Pergamon, 1971, vol. 2.

[35] P. B. Corkum, N. H. Burnett, and F. Brunel, "Multiphoton ionization in large ponderomotive potentials," in Atoms in Intense Fields, M. Gavrila, Ed. New York: Academic, 1992, supplement 1, pp. 109-137.

[36] P. H. Bucksbaum, M. Bashkansky, and T. J. Mcllrath, "Scattering of electrons by intense coherent light," Phys. Rev. Lett., vol. 58, pp. 349-352, 1987.

[37] T. J. Englert and E. A. Rinehart, "Second-harmonic photons from the interaction of free electrons with intense laser radiation," Phys. Rev. A, vol. 28 , pp. $1539-1545,1983$.

[38] J. Schwinger, "The quantum correction in the radiation by energetic accelerated electrons," in Proc. Nat. Acad. Sci. USA, 1954, vol. 40, pp. 132-136.

[39] H. R. Reiss, "Production of electron pairs from a zero-mass state," Phys. Rev. Lett., vol. 26, pp. 1072-1075, 1971.

[40] D. D. Meyerhofer, J. P. Knauer, S. J. McNaught, and C. I. Moore, "Observation of relativistic mass shift effects during high-intensity, laser-electron interactions," J. Opt. Soc. Amer. B, vol. 13, pp. 113-117, 1996.

[41] C. I. Moore, "Observation of the transition from Thomson to Compton scattering in optical multiphoton interactions with electrons," Ph.D. dissertation, Univ. Rochester, Rochester, NY, 1995.

[42] Y.-H. Chuang, D. D. Meyerhofer, S. Augst, H. Chen, J. Peatross, and S. Uchida, "Suppression of the pedestal in a chirped-pulse-amplification laser," J. Opt. Soc. Amer. B, vol. 8, pp. 1226-1235, 1991.

[43] S. Augst, D. Strickland, D. D. Meyerhofer, S. L. Chin, and J. H. Eberly, "Tunneling ionization of noble gases in a high-intensity laser fields," Phys. Rev. Lett., vol. 63, pp. 2212-2215, 1989.

[44] C. Bamber, T. Blalock, S. Boege, T. Kotseroglou, A. C. Melissinos, W. Ragg, D. Reis, D. D. Meyerhofer, J. Kelly, and M. Shoup, III, "0.5-Hz, phase-stabilized terawatt laser system with a Nd:glass slab amplifier for nonlinear QED experiments," Laser Phys., vol. 7, pp. 135-140, 1997.

[45] T. Kotseroglou, C. Bamber, S. Boege, A. C. Melissinos, D. D. Meyerhofer, W. Ragg, C. Bula, K. T. McDonald, E. J. Prebys, D. Berstein, D. Burke, E. Cisneros, R. C. Field, G. Horton-Smith, K. Jobe, J. Judkins, A. C. Odian, M. Ross, D. Waltz, S. C. Berridge, W. M. Bugg, K. Shmakov, and A. W. Weidemann, "Picosecond timing of terawatt laser pulses with the SLAC $46 \mathrm{GeV}$ electron beam," Nucl. Instrum. Methods Phys. Res. A, vol. 383, pp. 309-317, 1996.

[46] D. L. Burke, R. C. Field, G. Horton-Smith, J. E. Spencer, D. Walz, S. C. Berridge, W. M. Bugg, K. Shmakov, A. W. Wiedemann, C. Bula, K. T. McDonald, E. J. Prebys, C. Bamber, S. J. Boege, T. Koffas, T. Kotseroglou, A. C. Melissinos, D. D. Meyerhofer, D. A. Reis, and W. Ragg, "Positron production in multiphoton light-by-light scattering," Phys. Rev. Lett., vol. 79, pp. 1626-1629, 1997.

David. D. Meyerhofer, photograph and biography not available at the time of publication. 